\title{
Continuity of care for people with psychotic illness: its relationship to clinical and social functioning.
}

Article

Accepted Version

Catty, J., White, S., Clement, S., Cowan, N., Geyer, C., Harvey, K., Rees Jones, I., McLaren, S., Poole, Z., Rose, D., Wykes, T. and Burns, T. (2013) Continuity of care for people with psychotic illness: its relationship to clinical and social functioning. International Journal of Social Psychiatry, 59 (1). pp. 5-17. ISSN 1741-2854 doi:

https://doi.org/10.1177/0020764011421440 Available at https://centaur.reading.ac.uk/31091/

It is advisable to refer to the publisher's version if you intend to cite from the work. See Guidance on citing.

To link to this article DOI: http://dx.doi.org/10.1177/0020764011421440

Publisher: Sage

All outputs in CentAUR are protected by Intellectual Property Rights law, including copyright law. Copyright and IPR is retained by the creators or other copyright holders. Terms and conditions for use of this material are defined in the End User Agreement. 


\section{CentAUR}

Central Archive at the University of Reading

Reading's research outputs online 


\section{Continuity of care for people with psychotic illness: its relationship to clinical and social functioning}

[Continuity of care for psychotic illness]

Jocelyn Catty $^{1}$, Sarah White ${ }^{1}$, Sarah Clement ${ }^{2}$, Naomi Cowan ${ }^{1}$, Connie Geyer ${ }^{1}$, Kate Harvey ${ }^{3}$, Ian Rees Jones ${ }^{4}$, Susan McLaren ${ }^{5}$, Zoe Poole ${ }^{1}$, Diana Rose ${ }^{2}$, Til Wykes $^{2}$ and Tom Burns ${ }^{6}$ for the ECHO Group

Word count: 4,578

Abstract: 150

\section{The ECHO Group:}

Main Phase: Tom Burns ${ }^{6}$, Jocelyn Catty ${ }^{1}$, Sarah Clement ${ }^{2}$, Kate Harvey ${ }^{3}$, Sarah White $^{1}$, Tamara Anderson ${ }^{1}$, Naomi Cowan ${ }^{1}$, Gemma Ellis ${ }^{1}$, Helen Eracleous ${ }^{1}$, Connie Geyer $^{1}$, Pascale Lissouba ${ }^{1}$, Zoe Poole ${ }^{1}$;

Qualitative Strand: Ian Rees Jones ${ }^{4}$, Nilufar Ahmed ${ }^{1}$;

Developmental Phase: Diana Rose ${ }^{2}$, Til Wykes ${ }^{2}$, Angela Sweeney ${ }^{2}$;

Organisational Strand: Susan McLaren ${ }^{5}$, Ruth Belling ${ }^{5}$, Jonathon Davies ${ }^{5}$, Ferew Lemma $^{5}$, Margaret Whittock ${ }^{1}$.

1. St. George's, University of London, Cranmer Terrace, London SW17 0RE.

2. Institute of Psychiatry, De Crespigny Park, PO Box 77, London SE5 8AF.

3. University of Reading, School of Psychology, Earley Gate, Whiteknights, PO Box 238, Reading RG6 6AL.

4.School of Social Sciences, University of Wales, Bangor, Wales.

5. Institute of Primary Care and Public Health, London South Bank University, 103 Borough Road, London SE1 OAA.

6. For correspondence: Department of Psychiatry, University of Oxford, Warneford Hospital, Oxford OX3 7JX. Tel: + 44 (0)1865 226474. < tom.burns@psych.ox.ac.uk> 


\begin{abstract}

\section{Background}

The relationship between continuity of care and user characteristics or outcomes has rarely been explored. The ECHO Study operationalised and tested a multi-axial definition of continuity of care, producing a seven-factor model utilised here.
\end{abstract}

\title{
Aims
}

To assess the relationship between user characteristics and established components of continuity of care, and the impact of continuity on clinical and social functioning.

\section{Methods}

180 CMHT users with psychotic disorders were interviewed at three annual timepoints, assessing their experiences of continuity of care and clinical and social functioning. Scores on seven continuity factors were tested for association with userlevel variables.

\section{Results}

Improvement in quality of life was associated with better Experience \& Relationship continuity scores (better user-rated continuity therapeutic relationship) and with lower Meeting Needs continuity factor scores. Higher Meeting Needs scores were associated with a decrease in symptoms.

\section{Conclusion}

Continuity is a dynamic process, influenced significantly by care structures and organisational change.

\section{Keywords}

Continuity of care; severe mental illness; community care. 


\section{Background}

Continuity of care is considered essential (Crawford et al., 2004) but until recently has rarely been defined, while its specific meaning for people with severe mental illness has been unclear. Its relationship to user characteristics and outcomes has also rarely been explored. Mental health studies have generally either examined outcomes with implications for continuity or assessed interventions assumed to promote continuity (analysing other outcomes, such as clinical outcomes and satisfaction) (Freeman et al., 2000). Thus Killaspy and colleagues (2000) proposed that gaps in outpatient care were more likely to lead to hospital admission, and Bassett and colleagues (2000) found that poor continuity of staff and services following hospital discharge led to more rapid readmission; while Wasylenki and colleagues (1985) compared case management to a control service and found no difference in social functioning or symptomatology. Olfson and colleagues (1998) found that communication with the prospective outpatient clinician before discharge was linked with better mental health outcomes. Links between continuity of care and satisfaction have also been explored (Fan et al., 2004; Saultz \& Albedaiwi, 2004), as well as between poor continuity and suicide (Appleby et al., 1999; Desai et al., 2005). While transitions in care or hospital discharge have frequently been the focus of continuity research (Greenberg \& Rosenheck, 2005; Herman et al., 2000; Olfson et al., 1998), how continuity of care might impact on outcomes for users living in the community and in need of stable care has rarely been considered.

Freeman and colleagues (2000) proposed a multi-axial definition of continuity, comprising: 'experienced' ( 'experience of a coordinated and smooth progression of care from the user's point of view'), 'flexible' ('flexible and adjust[ing] to the needs of the individual over time'), 'relational' ('one or more named individual professionals with whom the user can establish and maintain a therapeutic relationship'), 'cross-boundary' ('effective communication between professionals and services and with service users'), 'longitudinal' ('care from as few professionals as possible, consistent with other needs') and 'information' continuity ('excellent information transfer following the user'). They subsequently added 'contextual' ('sustain a person's preferred social and personal relationship in the community and 
enhance quality of life') and 'long-term' continuity ('uninterrupted care for as long as the service user requires it') for mental health service users (Freeman et al., 2002).

The ECHO study was designed to explore the significance of continuity of care for people with mental health problems. It took Freeman and colleagues' multi-axial model as its theoretical starting-point and established from patient-level data that this model could be successfully operationalised for this group (Burns et al., 2009). 'Experienced continuity' from the original model was interpreted as an over-arching concept reflecting the user perspective and was operationalised in a user-rated measure, developed by service users for the study (Rose et al., 2009). The remaining definitions were operationalised using a range of data on service use and patterns of contact, collected from service users and records, along with data from two self-report measures, the Camberwell Assessment of Need (CAN: Phelan et al., 1996) and the Scale to Assess Therapeutic Relationships in Community Mental Health Care service user version (STAR: McGuire-Snieckus et al., 2007).

Factor analysis established seven distinct factors, confirming that continuity of care is a multi-factorial concept (Burns et al., 2009). These factors differed from the original elements of the theoretical model. Box 1 shows each factor, the components loading onto it and a definition based on our interpretation. The factor names were selected as the 'best fit' for the components loading onto each factor in the factor analysis, and are not always intuitive. The factors, rather than the definitions in the original model, were used for all subsequent analyses.

\section{- Box 1 about here -}

The current paper reports the main findings of the study concerning the relationships between continuity of care, user characteristics and clinical and social outcomes. The study was designed to allow both for continuity being multi-factorial and for the possibility of its either impacting upon clinical and social variables or being affected by them. 


\section{Objectives}

To assess the relationship between service user characteristics and continuity of care, and the impact of continuity on clinical and social functioning.

\section{Material and Methods}

\section{Sample, setting and procedure}

The study took place between 2002 and 2007, with recruitment to the phase reported here commencing in 2003. People with long-term psychotic disorders were sampled from the caseloads of seven community mental health teams (CMHTs) in two mental health Trusts. The inclusion criteria were: diagnosis of any psychotic disorder and in contact with psychiatric services for at least two years, on the CMHT caseload for at least six months, on the enhanced level of the Care Programme Approach (CPA, Department of Health, 1999) (indicating allocation to a key worker or case manager) and aged 18 to 65 . Recruitment was done on the basis of clinical diagnosis, but diagnoses were confirmed by use of OPCRIT (McGuffin et al., 1991), a validated structured assessment, by an independent clinically-trained researcher.

Interviews with users were conducted at baseline (T1) and at one- and two-year follow-up (T2 and T3), mapping the users' journeys through care in the year prior to interview. Data were collected on demographic and illness variables; patterns of contact with organisations and individual professionals; breaks in care; days in hospital; psychiatric symptoms (Brief Psychiatric Rating Scale, BPRS: Overall \& Gorham, 1976); functioning (Global Assessment of Functioning, GAF: Endicott et al., 1976); needs for care (CAN); therapeutic relationship (STAR); quality of life (Manchester Short Assessment of Quality of Life, MANSA: Priebe et al., 1999); Schedule for the Evaluation of Individual Quality of Life, SEIQoL: McGee et al., 1991); and empowerment (User Empowerment Scale: Rogers et al., 1997). CONTINU-UM was used to measure user-rated overall 'experienced' continuity. Quality of life was assessed using two measures to incorporate their different perspectives, MANSA being long-established as a validated measure in mental health while the more recent SEIQoL uses five domains suggested and weighted by the user. 
Data were also collected via interview on patterns of contact with organisations and individual professionals and on breaks in care, while data on contact with services, number of professionals and information flow were collected from CMHT records. All data related to continuity (such as service contact and information flow) were collected for the year prior to interview.

\section{Sample size}

The study was originally powered on the possibility of 31 variables being analysed as potential predictors of days in hospital. Using the estimate of 15 subjects required for each explanatory variable in the multi-level analysis gave a sample size of 465 . Because the variables were to be collected at repeated time-points, this sample size could be adjusted (Machin et al., 1997). Assuming a correlation between two observations made on the same subject of 0.6 (ibid.) and the study design allowing for data to be collected at three time-points, this gave an adjustment factor of 0.373. A sample size of $\left(465^{*} 0.373=\right) 174$ would therefore be sufficient. In practice, 21 variables were tested, ensuring that the analysis was adequately powered.

\section{Analysis}

The seven factors were scored using the components found to load onto them in our factor analysis (see Box 1), so that each user was given a factor score indicating their level of that factor at each time-point (Burns et al., 2009).

We conducted three analyses. The first two examined clinical and social variables associated with the continuity of care factors: Analysis 1 assessed associations between the variables as explanatory variables and the continuity factor scores as contemporaneous data-points, while Analysis 2 assessed associations between levels of the continuity factors and change in clinical and social functioning in the previous year. Analysis 3 explored the impact of continuity of care on clinical and social outcomes by assessing the association between the continuity factors and change in clinical and social variables over the subsequent year. Thus in the first analysis, the seven continuity factors were used as the outcome (dependent) variables, while in the second and third analyses they were used as possible explanatory (independent) variables. Each of these analyses was multi-level, using data from multiple timepoints. 


\section{User, care and illness characteristics associated with Continuity of Care}

Analysis 1: Contemporaneous associations with continuity of care factors

The following possible explanatory variables were tested against each continuity factor as the dependent variable: time-point, Trust, team, gender, total number of lifetime admissions, type of accommodation, living situation, ethnic group, education, employment, informal carer, use of depot medication, alcohol or drugs, whether hospitalised in the previous year, age, duration of illness, functioning, symptomatology, empowerment and quality of life. A multi-level model provided the framework for this analysis. This allowed the maximum use of all data, not requiring complete data on all variables at all time-points, while accounting for the dependent structure within the data (multiple time-points per subject).

For continuous dependent variables, the associations between each explanatory variable listed above and the continuity factor score were tested individually. PROC MIXED from SAS version 9.1 statistical software (SAS Institute Inc., 1989) was used, incorporating a random effect for service user. A final model for each continuity factor was then fitted, entering all explanatory variables which had been significantly related univariately $(\mathrm{p}<0.1)$. For each continuity factor, those explanatory variables found to be significant at the $10 \%$ level are presented with both their unadjusted parameter estimates (univariate analyses) and adjusted parameter estimates (multifactorial analyses). Continuous explanatory variables provide parameter estimates which indicate the rate of change of the variable in relation to the level of the continuity factor. For categorical explanatory variables, the estimated mean level of the continuity factor is presented for each category of the variable.

For the categorical factor Managed Transitions, multinomial logistic regression was carried out, using PROC GLIMMIX, incorporating a user random effect to allow for repeated measurements (Kuss \& McLerran, 2007) for trichotomous dependent variables. For these analyses, the parameter estimates are presented in the text below as odds ratios. 
Analysis 2: Associations between continuity factors and change in previous year

For the continuous dependent variables (one-year changes in clinical and social variables), the rating of the continuity factor utilised as the independent variable was that corresponding to the contemporaneous year. Thus the continuity scores for all seven factors at T2 were tested against change in each clinical and social variable between $\mathrm{T} 1$ and $\mathrm{T} 2$, while the continuity scores for all the factors at $\mathrm{T} 3$ were tested against change from T2 to T3 in the same variables. The same clinical and social variables were utilised as above.

As change variables were being used, the multiple measurements per user were not correlated, so a simple linear regression was used when testing the continuous continuity factors and a one-way analysis of variance when testing the categorical continuity factors. When 'hospitalised in the previous year' was the dependent variable, PROC GLIMMIX was used to incorporate a random user effect. Data from all three time-points were used in this analysis. Parameter estimates are presented for all independent variables significant at the $10 \%$ level and adjusted models were also fitted if more than one independent variable was significant.

\section{The impact of continuity of care on clinical and social outcomes (Analysis 3)}

This analysis was conducted as for Analysis 2, but to explore change in the subsequent year, each continuity factor at $\mathrm{T} 1$ was tested for association with the T1T2 change in each clinical and social variable and each continuity factor at T2 was tested for association with the T2-T3 change in each clinical and social variable, simultaneously. When hospitalisation (measured for the previous year at each datapoint) was the dependent variable, the continuity factor at $\mathrm{T} 1$ was tested for association with whether or not the service user had been hospitalised between $\mathrm{T} 1$ and $\mathrm{T} 2$ and the continuity factor at T2 was tested for association with whether or not the user had been hospitalised between $\mathrm{T} 2$ and $\mathrm{T} 3$.

\section{Results}

\section{Sample}

We approached 498 service users, of whom 318 declined to participate or were ineligible, leaving 180 (36\%) interviewed at T1 (85 from Trust 1, 95 from Trust 2). At 
T2, $165(91.7 \%)$ and at T3, 141 users (78.3\%) were interviewed, the remainder declining or being uncontactable. (Figure 1.)

- Figure 1 about here -

\section{Baseline characteristics, drop-out and discharge}

Table 1 shows the baseline data for the sample. Those who dropped out by T3 were significantly younger (mean age=39.46 years (SD: 12.37) versus 44.13 (SD: 10.28), $\mathrm{t}=2.397, \mathrm{p}=0.018$ ). They also had poorer quality of life (MANSA: 4.21 (SD: 0.97) versus 4.60 (SD: 0.76), $\mathrm{t}=2.661, \mathrm{p}=0.008)$, more symptoms (36.79 (SD: 11.95) versus 32.09 (SD: 10.45), t=-2.384, p=0.018) and lower Supported Living scores (-1.95 (SD: 1.23 ) versus $-1.20(\mathrm{SD}: 1.57), \mathrm{t}=2.740, \mathrm{p}=0.007)$. There were no other significant differences between the two groups.

By the end of the study, 41 service users $(22.8 \%)$ had been discharged from secondary care. They remained in the study unless lost to follow-up $(n=10)$. There were few significant differences between them and those who remained in secondary care, but the latter were more than twice as likely to have been on depot injections at T1 (51 $(37.5 \%)$ versus $\left.6(15.8 \%), \chi^{2}=6.36, p=0.012\right)$. Discharged users had slightly better functioning (mean (SD) GAF: 55.5 (17.3) vs 51.1 (13.6), t=-2.369, p=0.021) and fewer symptoms (mean (SD) BPRS: 31.3 (11.5) vs 33.3 (10.9), $\mathrm{t}=2.28, \mathrm{p}=0.025$ ) at T1. They had significantly lower Regularity scores (-0.56 (2.12) vs 0.37 (2.21), $\mathrm{t}=2.37, \mathrm{p}=0.019$ ), consistent with their being seen less often during the preceding year, but there were no other differences in factor scores.

- Table 1 about here -

User, care and illness characteristics associated with Continuity of Care Analysis 1: Contemporaneous associations with continuity of care factors

Each table (Tables 2-6) lists only those variables included in the multifactorial analysis, that is, those that were univariately associated with the continuity factor at the $p<0.1$ level. For categorical independent variables, values in the tables show the mean factor scores for each category in the variable; for continuous independent variables, they represent slope estimates (amount of change in the factor given a oneunit change in the variable). Values for the factor scores have no inherent meaning (a 
value of zero does not indicate having no continuity) and should only be interpreted relatively. Only the variables that remained in each model are discussed below, except where stated.

Users who scored highly on Experience \& Relationship were more likely to report better quality of life (MANSA) and also had higher factor scores at T2. (Table 2.) Users who scored highly on Regularity were more likely to be in Trust 1 and have depot injections, while higher scores on this factor also became more likely over time. (Table 3.) Users scoring highly on Meeting Needs were more likely to be living in supervised accommodation, have been ill for longer, have a lower level of functioning and more symptomatology and report poorer quality of life (MANSA). (Table 4.)

- Tables 2-4 about here -

Users who scored highly on Consolidation were more likely to be in Trust 2, or in one particular team from this Trust, or having depot injections. (Table 5.) Managed Transitions was related only to functioning, with a five-point higher GAF rating being associated with $10 \%$ lower odds of experiencing transitions. It was not possible to compare the levels of this factor for the documented and undocumented transitions groups directly as insufficient data meant that the regression models did not converge to provide parameter estimates. Users scoring highly on Care Coordination were more likely to have no identified informal carer. (Table 6.) Users scoring highly on Supported Living were more likely to be in Trust 1, have left school by 16 , live with others and have poorer functioning. (Table 6.)

- Tables 5 \& 6 about here -

\section{Analysis 2: Associations between continuity factors and change in previous year}

There was a considerable range of change scores for each clinical and social variable, although the mean changes were not of great magnitude. (Data available on request.)

A higher Experience \& Relationship score was associated with having experienced an improvement in quality of life during the previous year (SEIQoL: $\beta=1.4 ; 95 \% \mathrm{CI}$ : $0.59,2.3)$. A higher Meeting Needs score was associated with experiencing a deterioration in quality of life (MANSA: $\beta=-0.05 ; 95 \%$ CI: $-0.09 ;-0.01$ ) and an 
increase in symptomatology $(\beta=0.61 ; 95 \%$ CI: $0.10 ; 1.1)$ during the previous year. A higher Consolidation score and higher Care Coordination scores were each associated with lower odds of having been hospitalised in the previous year $(\mathrm{OR}=0.813,95 \% \mathrm{CI}$ : $0.674,0.981$; and $\mathrm{OR}=0.83,95 \% \mathrm{CI}: 0.68,1.02$, respectively), although the latter was only a weak association. Both factors retained similar significance when entered into the model together, suggesting that they are independent of each other. There were no other significant associations. It was not possible to estimate how Factor 5 (Managed Transitions) was related to hospitalisation, as all users who were hospitalised had experienced some form of transition, producing a non-estimable model.

\section{The impact of continuity of care on clinical and social outcomes (Analysis 3)}

Having a higher Meeting Needs score was associated with a decrease in symptomatology during the subsequent year $(\beta=-0.52 ; 95 \% \mathrm{CI}:-1.0 ;-0.1)$. Having a higher Experience \& Relationship score was associated with an increase in symptomatology during the subsequent year $(\beta=0.69 ; 95 \% \mathrm{CI}: 0.28 ; 1.1)$, as was having a higher Supported Living score $(\beta=0.89 ; 95 \%$ CI: $0.23 ; 1.6)$. Users with higher Regularity scores were more likely to be hospitalised in the subsequent year (OR: 1.166, 95\% CI: 0.977, 1.393). There were no other significant associations.

Box 2 shows a summary of each factor and the components associated with it in the multi-level analyses.

- Box 2 about here -

\section{Discussion}

User-level data utilised in this study demonstrates a range of relationships between user characteristics, experiences of services and the continuity of care factors which previous analyses (Burns et al., 2009) have established are meaningful for people with chronic psychotic disorders. This was an exploratory study and hence provides more evidence of associations than of causality; evidence of causal relationships is thus less meaningful than the network of associations that emerged from the data. Nevertheless, several key findings emerged. Higher Experience \& Relationship was associated with better quality of life, Regularity with having depot injections and 
being in Trust 1 and Meeting Needs with having a longer duration of illness, poorer functioning and quality of life, more symptoms and living in supervised accommodation. Higher Consolidation was associated with being in Trust 2 and having depot injections and Care Coordination with not having an informal carer, while lower odds of experiencing transitions was associated with higher functioning.

The names given to our continuity of care factors represent our interpretation of the components that loaded onto them in our previous analysis (Burns et al., 2009). They do not necessarily reflect best practice and in some cases are not intuitive to grasp. For instance, Care Coordination was the name given to the factor onto which loaded 'designated care coordinator', 'no designated psychiatrist' and 'fewer needs met by informal carers'. This suggested that when users had a care coordinator, it was not deemed so necessary in practice to ensure they could have a relationship with a single psychiatrist. Similarly, the loading of components onto Consolidation suggests that users whose care was more focused on one agency (namely, the CMHT) had less contact with primary care in practice. (For further discussion of this and the other factors, see Burns et al., 2009.)

\section{Limitations}

We were not able to measure any differential relationships between continuity of care and narrower diagnostic groups, such as bipolar disorder.

Those who dropped out were likely to be younger, have more symptoms and report lower quality of life and less likely to be in supported accommodation. It is difficult to assess the likelihood of response bias, but having more symptoms and lower quality of life were associated with having lower Experience \& Relationship scores. This suggests that the overall Experience \& Relationship scores, while not very high, may have been slightly inflated by the absence from analysis of those who had dropped out. Service users who refused to participate may also have been those who were less engaged with or favourably disposed towards services, which may have had the effect of lowering the reported levels of Experience \& Relationship and Regularity in particular. 
Changes in clinical and social functioning variables over time were not of great magnitude. This may be due to our having focused the study on a group expected to be in need of stable care, in order to detect meaningful discontinuities. Nevertheless, this may have limited the ability of the study to detect causal links between continuity factors and outcomes.

\section{Continuity of care factors and the service user characteristics associated with them}

It is unclear how Experience \& Relationship impacted upon clinical and social variables. Theoretically, better 'experienced continuity' and therapeutic relationship might be either a product of or give rise to better quality of life or fewer symptoms. Our analyses suggested that this factor was contemporaneously associated with better quality of life and with quality of life having improved over the previous year; but it was also associated with an increase in symptomatology over the subsequent year. Mood or general appraisal may have been a predictor of the components loading onto Experience \& Relationship; such a general appraisal factor may underlie a range of subjective user-rated outcome measures (Hansson et al., 2007, Priebe et al., 1998), including quality of life.

Service users with better functioning were less likely to have transitions than those with poorer functioning. The direction of effect is again unclear, as transitions in care might theoretically be a result or a cause of lower functioning. There was no evidence concerning any impact of change in the previous year, nor of the impact of transitions on change in the subsequent year.

Service users were more likely to have a higher Care Coordination score if they had no informal carer, suggesting that services may have taken into account their greater isolation or, conversely, that carers were filling a gap in professional input. Higher Care Coordination scores were also associated univariately with having depot injections, better functioning and less symptomatology and higher empowerment and quality of life (Table 6). There was no evidence of causality, but this may provide tentative evidence that care focused on a single care coordinator was having a positive impact on functioning and symptoms. Users who had a carer also scored higher on Experience \& Relationship and Regularity, although again this was only found in univariate analyses (Table 2). It may be that carers were play a mediating role with 
services, enabling users to access care (such as by encouraging attendance); conversely, those users who were less well functioning may have been both less able to maintain a relationship with a carer and correspondingly less able to engage with the services offered by professionals.

Users with high Supported Living scores had poorer functioning and were less educated, suggestive of a group of users who had been ill for more of their lives. Univariate analyses also suggested they were older and more chronically ill (Table 6). It is not surprising that this group needed the additional input of supported accommodation and day care. They experienced a worsening of symptoms over time, which was not accompanied by any increase in hospitalisation, suggesting that supported accommodation and day care may have obviated the need for hospitalisation that their worsening symptoms might otherwise have necessitated.

\section{A dynamic process}

Our analyses suggest that relationships between continuity of care factors and user characteristics are not uni-directional. On the contrary, key elements of continuity of care may be provided by professionals in response to specific user needs as these change, as well as impacting on them. Continuity of care is thus a dynamic process. This was particularly the case with the components involving different ratings of need. Having a high Meeting Needs score - indicating a high met need score as well as a high level of need - was followed by a decrease in symptomatology. This seems to demonstrate an interaction between professional inputs and user experiences, suggesting that treatment may be continually calibrated against need.

That services were picking up on the needs of those whose mental health was deteriorating may also be suggested by the fact that users with higher Regularity scores were more likely to be hospitalised in the subsequent year. This phenomenon may exemplify 'sensitive anticipatory casework' (Weaver et al., 2003), whereby professionals respond to users' needs to avert crisis. The extent to which this was successful for the service users in our study is unclear, however. While our analysis provides evidence of such dynamism, a sub-group interviewed in-depth (Jones et al., 2009) also felt that crises were not being sufficiently anticipated and that, on the contrary, they were invisible to services unless in crisis. These in-depth interviews 
also demonstrated clearly that continuity of care, particularly where it concerns userprofessional relationships, may be vulnerable to the impact of care transitions.

\section{Impact of differences between services}

In view of the need to enhance continuity of care for chronically ill populations, it is helpful to realise that, even with established disorders, healthcare organisation may have discernible effects at the user level. We found several aspects of continuity of care to be significantly different in practice between the two adjacent mental health Trusts, despite there being no differences in clinical and social functioning between service users in the two Trusts at T1.

Regularity was very much lower for users with psychotic disorders if they were in Trust 2. This is not surprising given that a related study of organisational practices (Burns et al., 2007) established that Trust 2 was going through a great deal of reorganisation at this time, with clear team-level effects (such as role change or resources) and that staff turnover and sickness were greater there. Indeed, at T1, 20 users $(22.2 \%)$ in Trust 2 reported that they had not seen a care coordinator in the previous year, unlike Trust 1 users who had all seen their care coordinator (Burns et al., 2007).

Staff turnover due to high use of agency, locum and bank staff (reported by staff in both Trusts) is also likely to have an adverse effect on therapeutic relationships (Belling et al., 2011). This would have a bearing on the factor Experience \& Relationship. Professionals in both Trusts also rated time for user contact inadequate due to administrative workloads (Belling et al., 2011). This too may partially account for the only moderate levels of therapeutic relationship reported. This is of particular concern not only because this was the factor accounting for most of the variance in the continuity data, but because users valued therapeutic relationships highly (Jones et al., 2009) and they have a known association with better outcomes (Catty, 2004). High nursing turnover is also known to have adverse effects on communication, engagement and medication management (Minore et al., 2005).

Thus although both Trusts had well-established routine CPA practices, apparently superficial differences in how services delivered the same model of care (here, 
generic CMHTs) had discernable impacts. The experiences of continuity of care reported here are clearly not intrinsic to the illness and attention to organisational aspects has real potential to improve users' experiences.

\section{Future research}

It is unclear whether the different facets of continuity are additive and further evidence is needed about which are more important to user experiences and outcomes. Further work is needed to identify the central continuity factors for establishing highquality care for people with chronic mental health problems. Serious consideration should also be given to including continuity of care routinely in service evaluations. With the increased emphasis on targeted services, exploration of the differential impacts of continuity of care factors on different diagnostic groups is clearly indicated.

\section{Acknowledgements}

This study was funded by a grant from the National Institute for Health Research Service Delivery and Organisation Programme (SDO13(d)/2001). Additional thanks are due to Dr Frank Holloway for his facilitation of this study and to Dr Andrew Molodynski for conducting clinical assessments of patient notes using OPCRIT. 


\section{References}

Appleby, L., Dennehy, J., Thomas, C., Faragher, E. \& Lewis, G. (1999) Aftercare and clinical characteristics of people with mental illness who commit suicide: a case-control study. Lancet, 353, 1397-1400.

Bassett, H., Lampe, J., Chris, L. (2000) Maintaining continuity of care. British Journal of Therapy and Rehabilitation, 7, 89-93.

Belling, R., Whittock, M., McLaren, S., Burns, T., Catty, J., Jones I.R., Rose, D., Wykes, T. \& the ECHO Group (2011) Achieving continuity of care: facilitators and barriers in community mental health teams. Implementation Science, 6, 23.

Burns, T., Catty, J., Clement, S., Harvey, K., Jones, I.R., McLaren, S., Rose, D., White, S. \& Wykes, T. for the ECHO Group (2007) Experiences of Continuity of Care and Health and Social Outcomes: The ECHO Study. Report to the National Coordinating Centre for Service Delivery and Organisation, London.

Burns, T., Catty, J., White, S., Clement, S., Ellis, G., Jones, I.R., Lissouba, P., McLaren, S., Rose, D. \& Wykes, T. for the ECHO Group (2009) Continuity of care in mental health: understanding and measuring a complex phenomenon. Psychological Medicine, 39, 313-323.

Catty, J. (2004) 'The vehicle of success': theoretical and empirical perspectives on the therapeutic alliance in psychotherapy and psychiatry. Psychology and Psychotherapy: Theory, Research and Practice, 77, 255-272.

Crawford, M., Jonge, E., Freeman, G. \& Weaver, T. (2004) Providing continuity of care for people with severe mental illness. Social Psychiatry and Psychiatric Epidemiology, 39, 265-272.

Department of Health (1999) A National Service Framework for Mental Health. London: HMSO.

Desai, R., Dausey, D. \& Rosenheck, R. (2005) Mental health service delivery and suicide risk: the role of individual patient and facility factors. American Journal of Psychiatry, 162, 311-318.

Endicott, J., Spitzer, R.L., Fleiss, J.L. \& Cohen, J. (1976) The Global Assessment Scale: A procedure for measuring overall severity of psychiatric disturbance. Arch Gen Psychiatry, 33, 766-771. 
Fan, V., Burman, M., McDonnell, M. \& Fihn, S. (2004) Continuity of care and other determinants of patient satisfaction with primary care. Journal of General Internal Medicine, 20, 226-233.

Freeman, G., Shepperd, S., Robinson, I., Ehrich, K. \& Richards S (2000) Continuity of Care: Report of a Scoping Exercise for the SDO Programme of NHS R\&D. London: National Coordinating Centre for Service Delivery and Organisation.

Freeman, G., Weaver, T., Low, J., de Jonge, E. \& Crawford, M. (2002) Promoting Continuity of Care for People with Severe Mental Illness whose needs span primary, secondary and social care. London: National Coordinating Centre for Service Delivery and Organisation.

Greenberg, G.A. \& Rosenheck, R.A. (2005) Continuity of care and clinical outcomes in a national health system. Psychiatric Services, 56, 427-33.

Hansson, L., Björkman, T. \& Priebe, S. (2007) Are important patient-rated outcomes in community mental health care explained by only one factor? Acta Psychiatrica Scandinavica, 116, 113-118.

Herman, D., Opler, L., Felxi, A., Valencia, E., Wyatt, R. \& Susser, E. (2000) A critical time intervention with mentally ill homeless men: impact of psychiatric symptoms. Journal of Nervous and Mental Disorder, 188, 135-140.

Jones, I.R., Ahmed, N., Catty, J., McLaren, S., Rose, D., Wykes, T. \& Burns, T. for the ECHO Group (2009) Illness careers and continuity of care in mental health services: a qualitative study of service user and carers. Social Science and Medicine, 69, 632-639.

Killaspy, H., Banerjee, S., King, M. \& Lloyd, M. (2000) Prospective controlled study of psychiatric out-patient non-attendance. British Journal of Psychiatry, 176, 160-165.

Kuss, O. \& McLerran, D. (2007) A Note on the Estimation of the Multinomial Logistic Model with Correlated Responses in SAS. Computer Methods and Programs in Biomedicine, 87, 262-269.

Machin, D., Campbell, M.J., Fayers, P.M., Pinol, A.P.Y. (1997) Sample size tables for clinical studies (ed 2). Oxford: Blackwell Science Ltd.

McGee, H.M., O'Boyle, C.A., Hickey, A., O’Malley, K. \& Joyce, C.R. (1991) Assessing the quality of life of the individual: the SEIQoL with a healthy and a gastroenterology unit population. Psychological Medicine, 21, 749-759. 
McGuffin, P., Farmer, A. \& Harvey, I. (1991) A polydiagnostic application of operational criteria in studies of psychotic illness. Development and reliability of the OPCRIT system. Archives of General Psychiatry, 48, 764-770.

McGuire-Snieckus, R., McCabe, R., Catty, J. \& Priebe, S. (2007) A New Scale to Assess the Therapeutic Relationship in Community Mental Health Care: STAR. Psychological Medicine, 37, 85-95.

Minore, B., Boone, M., Katt, M., Kinch, P., Birch, S. \& Mushquash, C. (2005) The effects of nursing turnover on continuity of care in isolated first nation communities. Canadian Journal of Nursing Research, 37, 87-100.

Olfson, M., Mechanic, D., Boyer, C., Hansell, S. (1998) Linking inpatients with schizophrenia to outpatient care. Psychiatric Services, 49, 911-917.

Overall, J.E. \& Gorham, D.R. (1976) Brief Psychiatric Rating Scale. In ECDEU Assessment Manual of Psychopharmacology (ed. Guy, W.), pp 157-169. Rockville, MD

Phelan, M., Slade, M., Thornicroft, G., Dunn, D., Holloway, F., Wykes, T., Strathdee, G., Loftus, L., McCrone, P. \& Hayward, P. (1996) The Camberwell assessment of need (CAN): the validity and reliability of an instrument to measure the needs of people with severe mental illness. British Journal of Psychiatry, 167, 589-595.

Priebe, S., Kaiser, W., Huxley, P. Rödder-Wanner, U-U. \& Rudolf, H. (1998) Do different subjective evaluation criteria reflect distinct constructs? Journal of Nervous and Mental Disorder, 186, 385-392.

Priebe, S., Huxley, P., Knight, S. \& Evans, S. (1999) Application and results of the Manchester Short Assessment of Quality of Life. International Journal of Social Psychiatry, 45, 7-12.

Rogers, E.S., Chamberlin, J., Ellison, M.L. \& Cream, T. (1997) A consumerconstructed scale to measure empowerment amongst users of mental health services. Psychiatric Services, 48, 1042-1047.

Rose, D., Wykes, T., Sweeney, A., Leese, M., Clement, S., Jones, I.R., Catty, J. \& Burns, T. for the ECHO Group (2009) Developing a user-generated measure of continuity of care. Acta Psychiatrica Scandinavica, 119, 320-324.

Saultz, J. \& Albedaiwi, W. (2004) Interpersonal continuity of care and patient satisfaction: a critical review. Annals of Family Medicine, 2, 445-451. 
Sytema, S., Micciolo, R. \& Tansella, M. (1997) Continuity of care for patients with schizophrenia and related disorders: a comparative South-Verona and Groningen case-register study. Psychological Medicine, 27, 1355-1362.

Wasylenki, D., Goering, P., Lancee, W., Ballantyne, R. \& Farkas, M. (1985) Impact of a Case Manager Program on Psychiatric Aftercare. Journal of Nervous and Mental Disorder, 173, 303-308.

Weaver, T., Tyrer, P., Ritchie, J. \& Renton, A. (2003) Assessing the value of assertive outreach: Qualitative study of process and outcome generation in the UK700 trial. British Journal of Psychiatry, 183, 437-445. 


\section{Tables \& Figures}

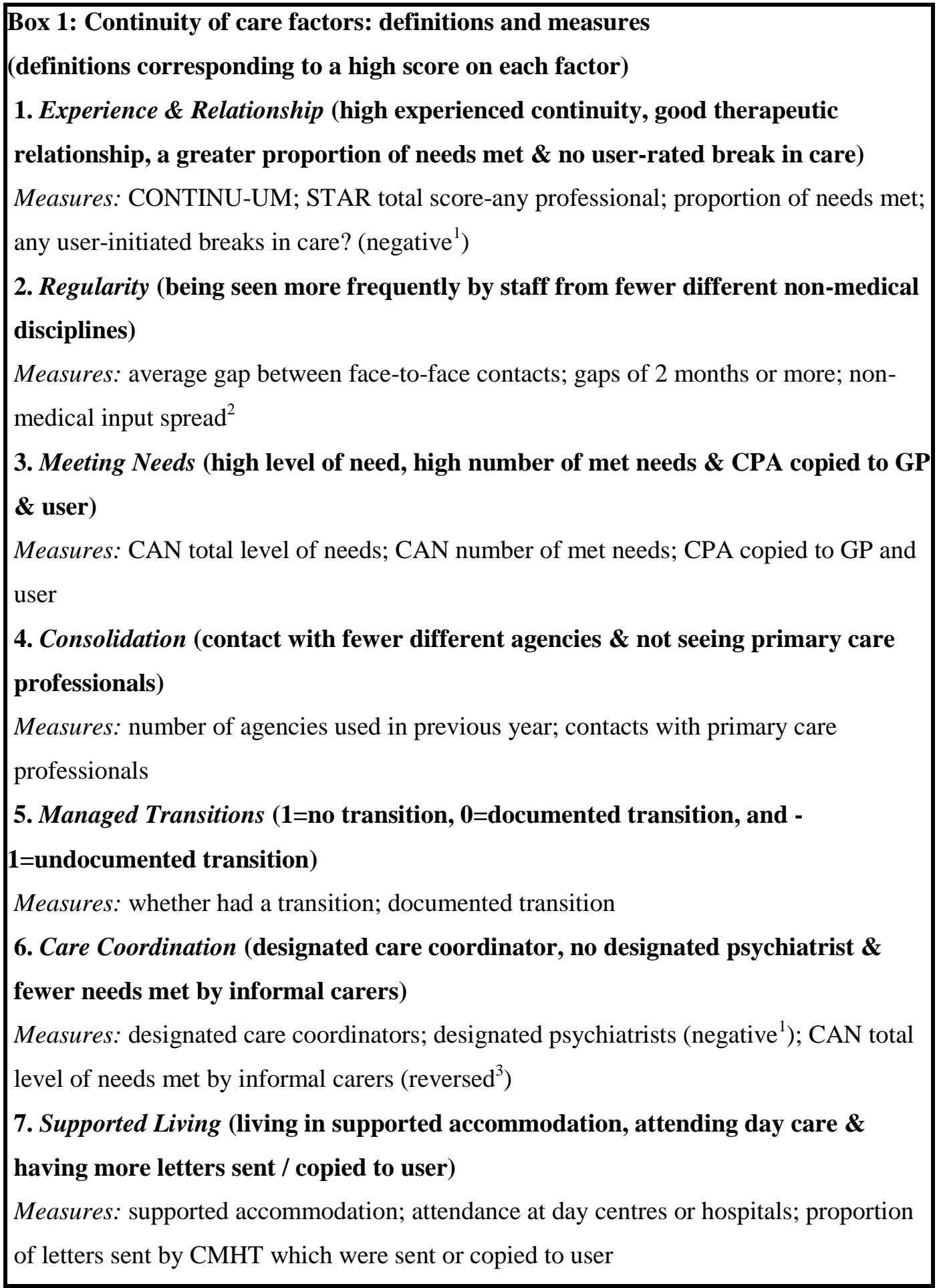

1.Component loads negatively on the factor, indicating an inverse relationship.

2. Number of non-medical professionals seen out of the total number of non-medical professionals in the team.

3. Variable was reverse-scored from the outset so that a high score would indicate a positive scenario. 
Figure 1: Recruitment and follow-up

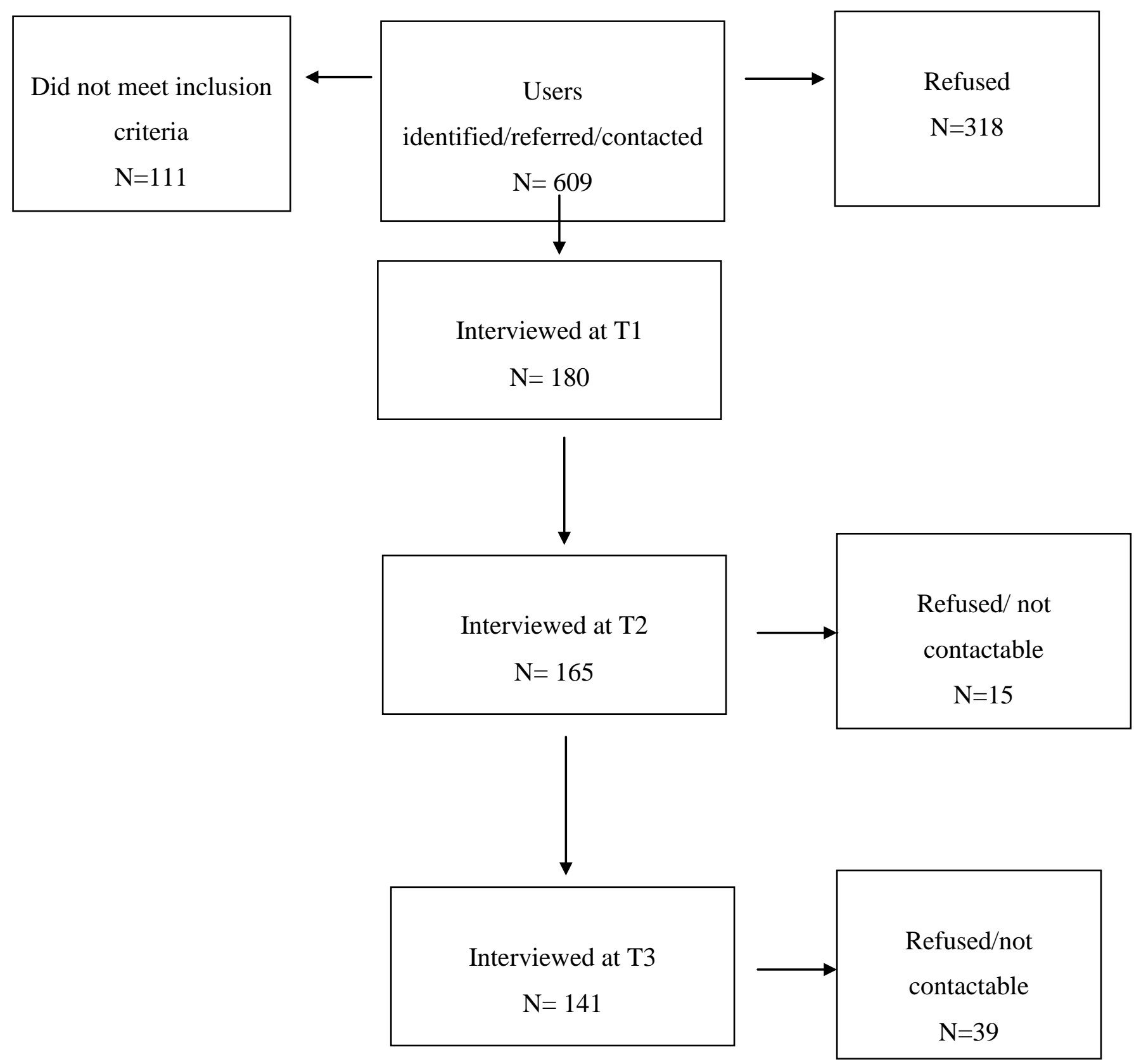




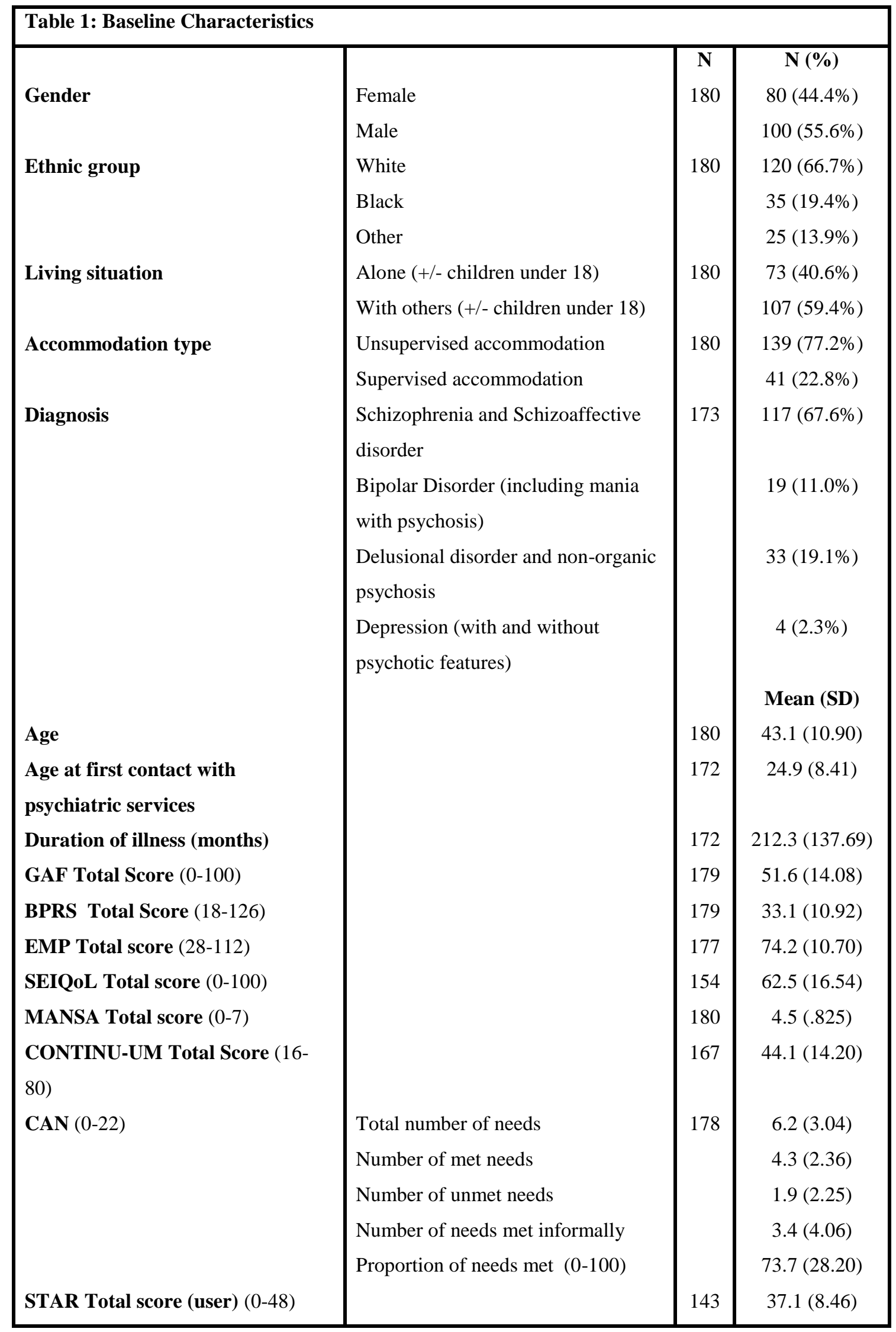


Table 2: Associations with FACTOR 1 - Experience \& Relationship

\begin{tabular}{|c|c|c|c|c|c|}
\hline & & Univari & model & $\overline{\text { Adjus }}$ & model \\
\hline Variable $^{1}$ & Label & Mean (se) & $95 \% \mathrm{CI}$ & Mean (se) & $95 \% \mathrm{CI}$ \\
\hline Time-point & $\mathrm{T} 1$ & $.93(.170)$ & $(.59,1.3)$ & $1.1(.183)$ & $(.71,1.4)$ \\
\hline & $\mathrm{T} 2$ & $2.6(.175)$ & $(2.2,2.9)$ & $2.7(.176)$ & $(2.3,3.0)$ \\
\hline & $\mathrm{T} 3$ & $.84(.186)$ & $(.47,1.2)$ & $.89(.188)$ & $(.52,1.3)$ \\
\hline Trust & 1 & $1.7(.204)$ & $(1.3,2.1)$ & $1.8(.197)$ & $(1.4,2.2)$ \\
\hline & 2 & $1.2(.193)$ & $(.84,1.6)$ & $1.3(.186)$ & $(.95,1.7)$ \\
\hline Living situation & $\begin{array}{l}\text { Living alone }(+/- \\
\text { children under } 18)\end{array}$ & $1.2(.208)$ & $(.80,1.6)$ & $1.3(.198)$ & $(.92,1.7)$ \\
\hline & $\begin{array}{l}\text { Living with others } \\
\text { (+/- children under } \\
\text { 18) }\end{array}$ & $1.7(.179)$ & $(1.3,2.0)$ & $1.8(.177)$ & $(1.4,2.1)$ \\
\hline Informal carer & Yes & $2.0(.238)$ & $(1.5,2.6)$ & $1.7(.218)$ & $(1.2,2.1)$ \\
\hline & No & $1.3(.156)$ & $(1.0,1.6)$ & $1.4(.145)$ & $(1.1,1.7)$ \\
\hline GAF & & $.03(.009)$ & $(.01, .05)$ & $.01(.011)$ & $(-.01, .03)$ \\
\hline BPRS & & $-.06(.011)$ & $(-.08,-.04)$ & $-.01(.014)$ & $(-.04, .01)$ \\
\hline Empowerment & & $.04(.010)$ & $(.02, .06)$ & $.01(.012)$ & $(-.01, .04)$ \\
\hline MANSA & & $1.0(.130)$ & $(.71,1.2)$ & $.59(.179)$ & $(.24, .95)$ \\
\hline SEIQoL & & $.04(.007)$ & $(.02, .05)$ & $.01(.008)$ & $\left(-.00^{2}, .03\right)$ \\
\hline
\end{tabular}

1. In the column for the adjusted model, variables significant at the 5\% level are shown in bold.

2. Lower $95 \%$ CI: -0.005 
Table 3: Associations with FACTOR 2 - Regularity

\begin{tabular}{|c|c|c|c|c|c|}
\hline & & Univaı & model & $\overline{\text { Adjus }}$ & model \\
\hline Variable $^{1}$ & Label & Mean (se) & $95 \% \mathrm{CI}$ & Mean (se) & $95 \% \mathrm{CI}$ \\
\hline Time-point & $\mathrm{T} 1$ & $.17(.160)$ & $(-.15, .49)$ & $.47(.165)$ & $(.15, .80)$ \\
\hline & $\mathrm{T} 2$ & $.66(.166)$ & $(.33, .98)$ & $.92(.166)$ & $(.59,1.2)$ \\
\hline & $\mathrm{T} 3$ & $.79(.168)$ & $(.46,1.1)$ & $1.1(.177)$ & $(.76,1.5)$ \\
\hline Trust & 1 & $1.3(.156)$ & $(1.0,1.7)$ & $1.6(.164)$ & $(1.3,1.9)$ \\
\hline & 2 & $-.25(.153)$ & $(-.55, .05)$ & $.08(.171)$ & $(-.26, .41)$ \\
\hline Team $^{1}$ & Team 1a & $1.5(.274)$ & $(.93,2.0)$ & $1.8(.286)^{2}$ & $(1.3,2.4)^{2}$ \\
\hline & Team $1 \mathrm{~b}$ & $1.7(.394)$ & $(.89,2.4)$ & $1.7(.382)^{2}$ & $(.99,2.5)^{2}$ \\
\hline & Team 1c & $1.3(.360)$ & $(.56,2.0)$ & $1.5(.360)^{2}$ & $(.83,2.2)^{2}$ \\
\hline & Team 1d & $1.1(.272)$ & $(.59,1.7)$ & $1.3(.275)^{2}$ & $(.77,1.9)^{2}$ \\
\hline & Team $2 \mathrm{a}$ & $.05(.220)$ & $(-.39, .48)$ & $.35(.237)^{2}$ & $(-.11, .82)^{2}$ \\
\hline & Team $2 b$ & $-.49(.337)$ & $(-1.2, .17)$ & $-.31(.353)^{2}$ & $(-1.0, .38)^{2}$ \\
\hline & Team 2c & $-.53(.273)$ & $(-1.1, .00)$ & $-.10(.280)^{2}$ & $(-.66, .45)^{2}$ \\
\hline Informal carer & Yes & $.84(.218)$ & $(.41,1.3)$ & $1.0(.198)$ & $(.62,1.4)$ \\
\hline & No & $.42(.137)$ & $(.15, .69)$ & $.66(.128)$ & $(.40, .91)$ \\
\hline Depot injections & Yes & $1.4(.192)$ & $(.99,1.8)$ & $1.3(.186)$ & $(.90,1.6)$ \\
\hline & No & $.12(.148)$ & $(-.17, .41)$ & $.40(.144)$ & $(.11, .68)$ \\
\hline
\end{tabular}

1. In the column for the adjusted model, variables significant at the 5\% level are shown in bold.

2. Estimates of all the variables in the adjusted model are reported for the model when variable team is omitted, (The perfect correlation between the variables 'Team' and 'Trust' makes a model with both spurious). 
Table 4: Associations with FACTOR 3 - Meeting Needs

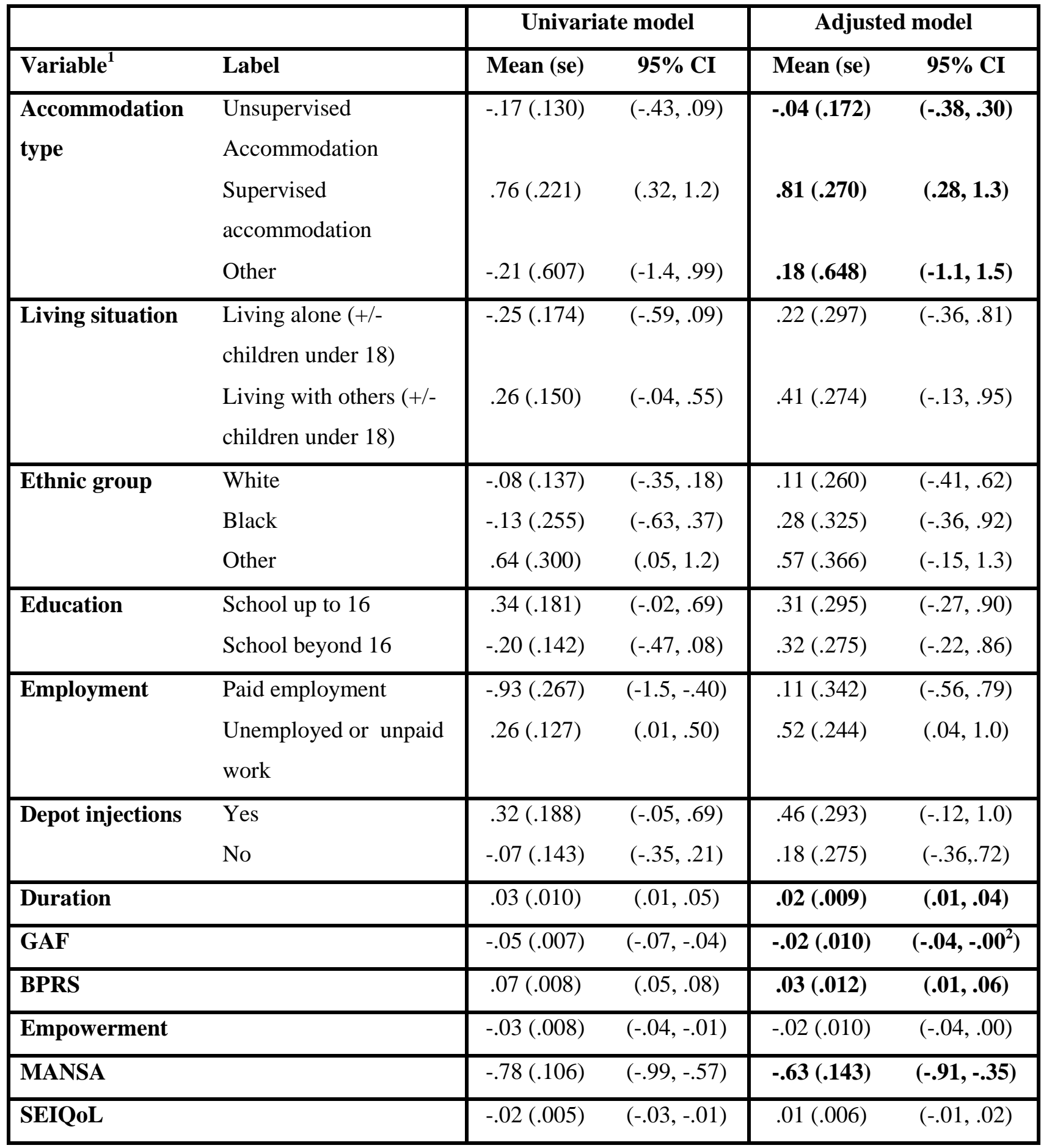

1. In the column for the adjusted model, variables significant at the 5\% level are shown in bold.

2. Upper $95 \%$ CI: -.00179 
Table 5: Associations with FACTOR 4 - Consolidation

\begin{tabular}{|c|c|c|c|c|c|}
\hline & & $\overline{\text { Univar }}$ & e model & $\overline{\text { Adjust }}$ & model \\
\hline Variable $^{1}$ & Label & Mean (se) & $95 \% \mathrm{CI}$ & Mean (se) & $95 \% \mathrm{CI}$ \\
\hline Trust & 1 & $-.16(.134)$ & $(-.43, .10)$ & $-.25(.164)$ & $(-.57, .08)$ \\
\hline & 2 & $.36(.127)$ & $(.11, .61)$ & $.41(.175)$ & $(.06, .75)$ \\
\hline Team $^{2}$ & Team 1a & $-.23(.240)$ & $(-.71, .24)$ & $-.30(.254)$ & $(-.80, .20)$ \\
\hline & Team $1 b$ & $-.38(.335)$ & $(-1.0, .28)$ & $-.45(.332)$ & $(-1.1, .21)$ \\
\hline & Team 1c & $.13(.318)$ & $(-.49, .76)$ & $.16(.326)$ & $(-.48, .80)$ \\
\hline & Team 1d & $-.15(.228)$ & $(-.60, .30)$ & $-.19(.241)$ & $(-.67, .28)$ \\
\hline & Team 2a & $.52(.188)$ & $(.15, .89)$ & $.40(.215)$ & $(-.02, .83)$ \\
\hline & Team 2b & $-.08(.296)$ & $(-.66, .50)$ & $.14(.315)$ & $(-.48, .76)$ \\
\hline & Team 2c & $.38(.213)$ & $(-.04, .80)$ & $.59(.245)$ & $(.10,1.1)$ \\
\hline Age & & $.02(.009)$ & $(.01, .04)$ & $.01(.012)$ & $(-.02, .03)$ \\
\hline$\overline{\operatorname{Sex}}$ & Male & $-.05(.125)$ & $(-.30, .20)$ & $-.01(.159)$ & $(-.32, .31)$ \\
\hline & Female & $.32(.140)$ & $(.04, .59)$ & $.17(.174)$ & $(-.18, .51)$ \\
\hline Education & School up to 16 & $.39(.151)$ & $(.10, .69)$ & $.19(.188)$ & $(-.18, .56)$ \\
\hline & School beyond 16 & $-.06(.118)$ & $(-.29, .17)$ & $-.03(.153)$ & $(-.33, .27)$ \\
\hline Duration & & $.02(.008)$ & $(.01, .04)$ & $.00(.001)$ & $\left(-.00^{4}, .00\right)$ \\
\hline Depot injections & Yes & $.43(.150)$ & $(.13, .72)$ & $.38(.189)$ & $(.01, .76)$ \\
\hline & No & $-.09(.113)$ & $(-.31, .13)$ & $-.22(.149)$ & $(-.52, .07)$ \\
\hline$\overline{\text { GAF }}$ & & $.01(.007)$ & $\left(-.00^{3}, .02\right)$ & $.01(.009)$ & $(-.01, .03)$ \\
\hline BPRS & & $-.02(.008)$ & $(-.04,-.01)$ & $-.01(.011)$ & $(-.03, .02)$ \\
\hline MANSA & & $.28(.097)$ & $(.09, .47)$ & $.09(.137)$ & $(-.18, .36)$ \\
\hline SEIQoL & & $.01(.005)$ & $(.00, .02)$ & $.01(.006)$ & $(-.01, .02)$ \\
\hline Hospitalised & Yes & $-.28(.223)$ & $(-.72, .16)$ & $-.01(.239)$ & $\overline{(-.48, .46)}$ \\
\hline & No & $.17(.098)$ & $(-.02, .37)$ & $.17(.105)$ & $(-.03, .38)$ \\
\hline
\end{tabular}

1. In the column for the adjusted model, variables significant at the 5\% level are shown in bold.

1. Estimates in adjusted model are reported for the model when variable Team is omitted. (The perfect correlation between the variables 'Team' and 'Trust' makes a model with both spurious).

2. Lower $95 \%$ CI: -.00177

3. Lower $95 \%$ CI: -.00098 
Table 6: Associations with FACTORS 6 \& 7 - Care Coordination \& Supported Living

\begin{tabular}{|c|c|c|c|c|c|}
\hline \multicolumn{2}{|c|}{ Care Coordination } & \multicolumn{2}{|c|}{ Univariate model } & \multicolumn{2}{|c|}{ Adjusted model } \\
\hline Variable $^{1}$ & Label & Mean (se) & $95 \%$ CI & Mean (se) & $95 \%$ CI \\
\hline \multirow[t]{2}{*}{ Informal carer } & Yes & $-.47(.149)$ & $(-.76,-.18)$ & $-.55(.148)$ & $(-.85,-.26)$ \\
\hline & No & $.17(.097)$ & $(-.02, .36)$ & $.21(.098)$ & $(.02, .40)$ \\
\hline \multirow[t]{2}{*}{ Depot injections } & Yes & $.24(.144)$ & $(-.04, .52)$ & $-.03(.145)$ & $(-.32, .25)$ \\
\hline & No & $-.12(.108)$ & $(-.33, .09)$ & $-.31(.107)$ & $(-.52,-.10)$ \\
\hline \multicolumn{2}{|l|}{$\overline{\text { GAF }}$} & $.01(.006)$ & $(.00, .03)$ & $.01(.008)$ & $\left(-.00^{3}, .03\right)$ \\
\hline \multicolumn{2}{|l|}{ BPRS } & $-.02(.007)$ & $(-.04,-.01)$ & $-.02(.010)$ & $(-.03, .00)$ \\
\hline \multicolumn{2}{|l|}{ Empowerment } & $.02(.006)$ & $(.01, .03)$ & $-.00^{4}(.008)$ & $(-.02, .01)$ \\
\hline \multicolumn{2}{|l|}{ MANSA } & $.32(.090)$ & $(.14, .49)$ & $.17(.118)$ & $(-.06, .41)$ \\
\hline \multicolumn{2}{|l|}{ SEIQoL } & $.01(.004)$ & $(.00, .02)$ & $.01(.005)$ & $\left(-.00^{5}, .02\right)$ \\
\hline \multicolumn{6}{|l|}{ Supported Living } \\
\hline \multirow[t]{3}{*}{ Time-point } & $\mathrm{T} 1$ & $-1.4(.113)$ & $(-1.6,-1.1)$ & $-1.4(.118)$ & $(-1.6,-1.1)$ \\
\hline & $\mathrm{T} 2$ & $-.95(.113)$ & $(-1.2,-.73)$ & $-1.1(.123)$ & $(-1.3,-.81)$ \\
\hline & $\mathrm{T} 3$ & $-.49(.113)$ & $(-.71,-.26)$ & $-.67(.130)$ & $(-.93,-.42)$ \\
\hline \multirow[t]{2}{*}{ Trust } & 1 & $-.77(.118)$ & $(-1.0,-.54)$ & $-.82(.131)$ & $(-1.1,-.56)$ \\
\hline & 2 & $-1.1(.112)$ & $(-1.3,-.86)$ & $-1.2(.120)$ & $(-1.5,-1.0)$ \\
\hline \multirow[t]{2}{*}{ Living situation } & Alone $^{2}$ & $-1.3(.132)$ & $(-1.6,-1.1)$ & $-1.2(.131)$ & $(-1.5,-.98)$ \\
\hline & $\begin{array}{l}\text { With } \\
\text { others }^{2}\end{array}$ & $-.89(.114)$ & $(-1.1,-.67)$ & $-.81(.114)$ & $(-1.0,-.59)$ \\
\hline \multirow[t]{3}{*}{ Education } & To 16 & $-.64(.129)$ & $(-.90,-.39)$ & $-.82(.147)$ & $(-1.1,-.54)$ \\
\hline & Beyond & $-1.1(.102)$ & $(-1.3,-.91)$ & $-1.2(.111)$ & $(-1.4,-1.0)$ \\
\hline & 16 & & & & \\
\hline \multicolumn{2}{|l|}{$\overline{\text { Age }}$} & $.01(.007)$ & $\left(-.00^{6}, .03\right)$ & $.01(.011)$ & $(-.01, .03)$ \\
\hline \multicolumn{2}{|l|}{ Duration } & $.02(.007)$ & $(.00, .03)$ & $.00(.011)$ & $(-.02, .02)$ \\
\hline \multicolumn{2}{|l|}{$\overline{\text { GAF }}$} & $-.02(.006)$ & $(-.03,-.01)$ & $-.01(.007)$ & $\left(-.03,-.00^{7}\right)$ \\
\hline \multicolumn{2}{|l|}{ Empowerment } & $-.01(.007)$ & $(-.03, .00)$ & $-.01(.008)$ & $(-.02, .01)$ \\
\hline
\end{tabular}

1. In the column for the adjusted model, variables significant at the 5\% level are shown in bold.

2. With or without dependent children.

3. Lower $95 \%$ CI: -0.00470

4. Mean: -0.00159

5. Upper $95 \%$ CI: -0.00449

6. Lower $95 \%$ CI: -0.00025

7. Upper $95 \%$ CI: -0.00095 
Box 2: Summary of continuity factors and components associated with them ${ }^{1}$

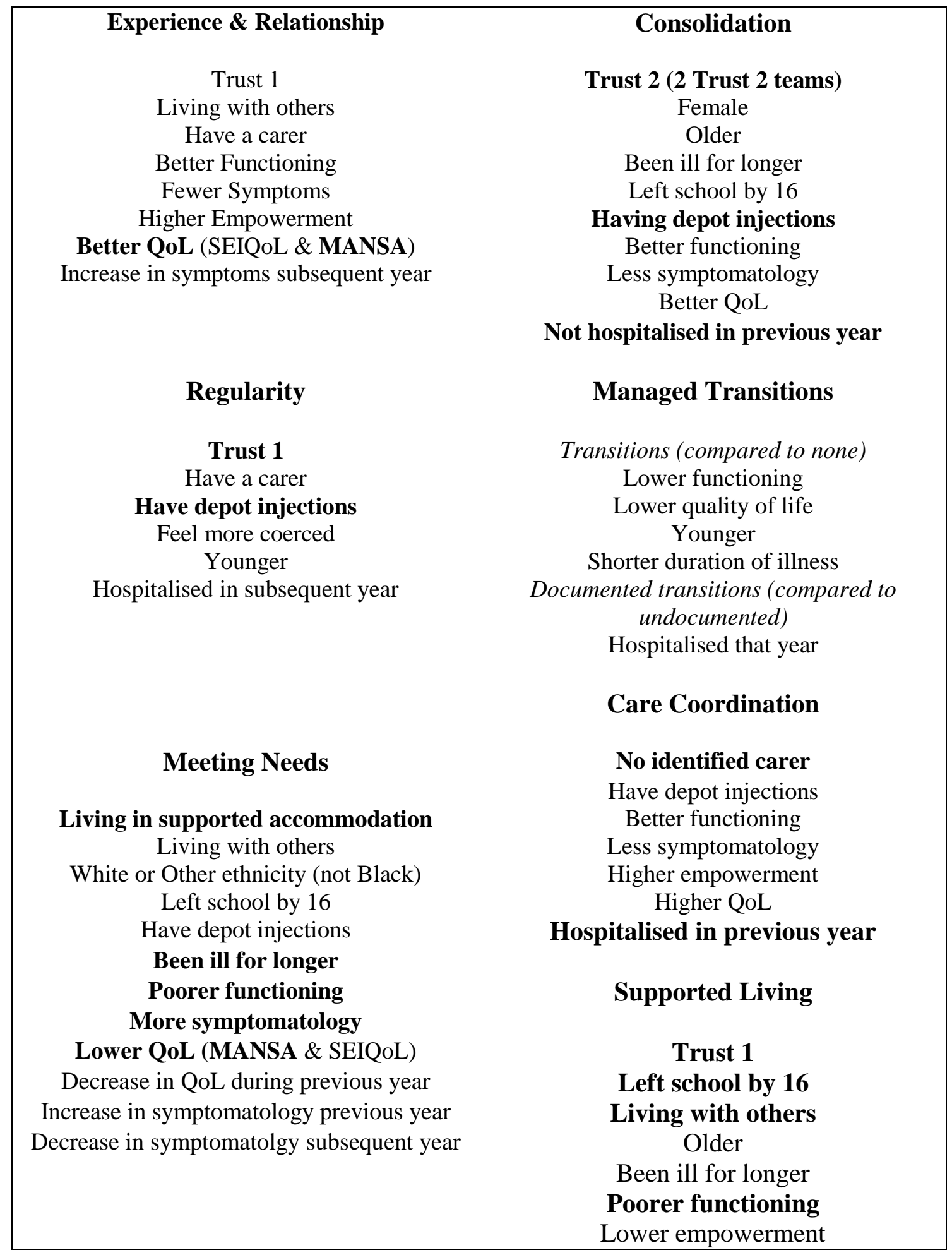

1. Data on time-point not shown here. Components in bold are those retained in the multi-level model. 